- Fracture of an implant is rare, occurring in about $0.6 \%$ of cases.

- Occlusal overload has been suggested as a cause.

- In this case the occlusal load was increased and fracture then occurred.

- Bridges with cantilever design on single implants will further increase bending forces on implants and therefore the supporting bone. This may lead to marginal bone loss.

- A treatment option leaving the implant fragment in situ is described.

\title{
Fracture of the body of an implant and its management - a case history
}

\author{
K. Gibney ${ }^{1}$
}

The increasing use of endosseous osseointegrated implants to replace natural teeth will inevitably lead to an increase in patients presenting in general dental practice with failing implants. A fractured implant body is not a common cause of failure, however it is a common cause of late failure. There is evidence that careful treatment planning can reduce the incidence of fracture. This paper describes a case demonstrating the management of implant fracture and discussing a possible mechanism for this failure. Some of the previously described factors that have been thought to contribute towards fracture are also discussed.

Endosseous osseointegrated implants are now widely used to replace missing teeth and there is a great deal of evidence to support their efficacy. ${ }^{1,2}$ Failures however do occur and although the most common cause is failure of integration, a less common cause is fracture of the body of the implant accounting for about $0.6 \%$ of failures. ${ }^{3}$

\section{CASE STUDY}

A 64-year-old female attended the surgery complaining of a loose and painful bridge at UR45 $(14,15)$. She gave a history of having an implant placed in this area about 2 years previously and more recently, 6 months ago, the crown at UR3 (13) had become de-cemented. After its re-cementation her bite had 'felt different'. Examination revealed an implantretained cantilever bridge at UR45 $(14,15)$ supported by a single implant at UR4 (14).

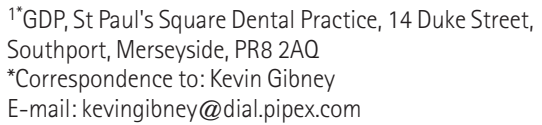

\section{Refereed Paper}

doi:10.1038/sj.bdj.4811824

Received 14.08.03; Accepted 19.01.04

( ) British Dental Journal 2004; 197: 615-617

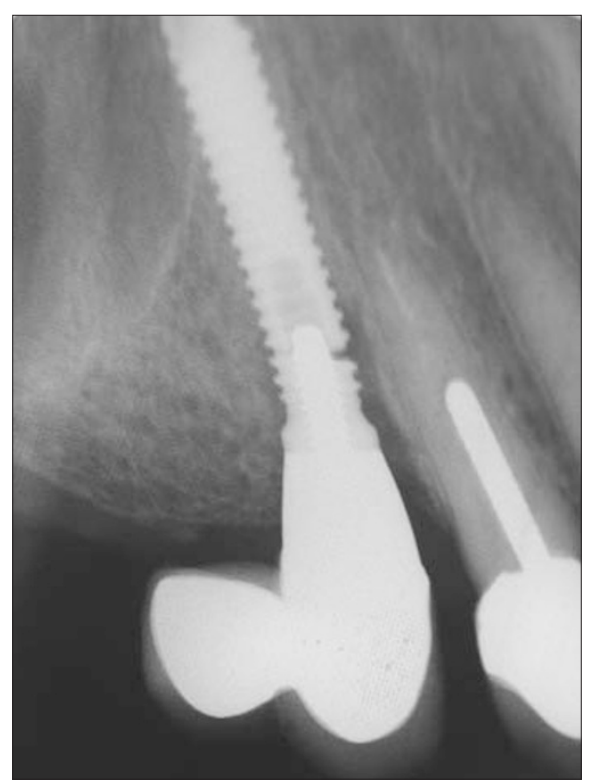

Fig. 1 Initial periapical radiograph showing fracture

The implant was a Brånemark Mark II (Nobel Biocare, Gothenburg, Sweden), regular platform $(3.75 \mathrm{~mm})$ fixture and was $18 \mathrm{~mm}$ in length. There was a postretained crown at UR3 (13). Some redness and swelling in the soft tissues around the implant was evident and the bridge was mobile. Teeth present on examination were UL2347 (22-24, 27), UR123 (11-13) and LL12345 (31-35) and LR1234578 (41-45, 47, 48). Fixed bridges were present replacing UL1 $(21)$ and $\operatorname{UL56}(25,26)$ with full crown retainers at UR1 (11), UL2 (22) and UL4 (24) and UL7 (27) respectively. LR6 (46) space was closed by drifting of LR78 $(47,48)$. All teeth present were symptom free.

A periapical radiograph (Fig. 1) revealed a fracture in the body of the implant at the position of the end of the prosthetic screw. The loose bridge was removed under local analgesia together with the implant fragment (Fig. 2) and a panoral radiograph was taken (Fig. 3).

A number of options were considered:

\section{Removable partial denture}

The patient did not want a removable solution therefore Option 1 was not considered further.

\section{Restoration of remaining implant fragment}

There was sufficient remaining implant (about $10 \mathrm{~mm}$ ) and prosthetic screw hole to support a single tooth restoration. This would have involved the preparation of the 
fragment to provide a flat prosthetic table and the construction of a customised abutment. The likelihood of failure was considered to be too high and as the patient also wanted the space at UR5 (15) restoring this option was not considered further.

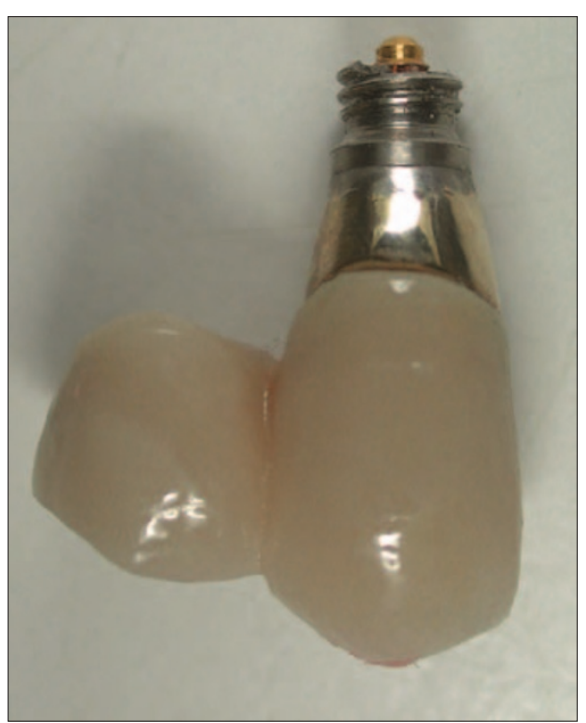

Fig. 2 Bridge and fixture fragment after removal

\section{Removal of fragment and new implant placed at site}

It was decided that the removal of the remaining implant fragment using a trephine would have resulted in considerable bone loss as a $4 \mathrm{~mm}$ trephine with external dimensions of $4.5 \mathrm{~mm}$ would have been used. It is likely that the placement of a second fixture at the same site would have required bone grafting to provide an adequate quantity of bone and this was not acceptable to the patient.

\section{Removal of UR3 (13) and implants placed at UR3 (13) and UR5 (15)}

This option was chosen as being most likely to provide a long-term stable solution that was acceptable to the patient. Two implants can be safely used to support a three-unit bridge to replace UR345 (13-15) and UR3 (13) was a failing post crown with a poor prognosis.

An immediate, temporary, removable partial denture was constructed to replace UR345 and UR3 was extracted. About 6 weeks later two implants were placed at UR3 and UR5 using the standard Brånemark protocol. They were both Brånemark implants being Mark IV TiUnite ${ }^{\circledR}$ implants of $15 \mathrm{~mm}$ and $11.5 \mathrm{~mm}$ lengths respectively. As the implant fragment was not visible a guide pin was screwed into the remaining implant fragment to mark its position during surgery (Fig. 4). A two-stage approach was to be used and therefore cover screws were placed and the wound closed. After

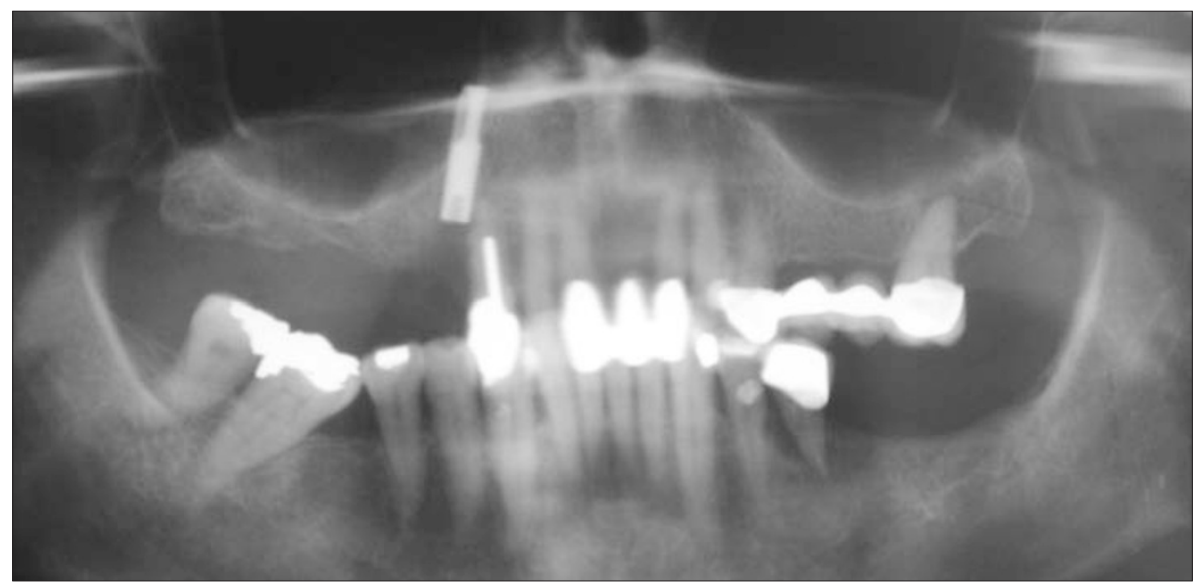

Fig. 3 Initial panoral radiograph showing remaining fixture fragment

6 months the implants were exposed and healing abutments attached.

The remaining implant fragment was below the crest of the bone and so it is likely that the bone would remodel around this possibly covering it completely. The full thickness flap raised for the placement of the implants was closed over the site and as can be seen in Figure 5 healing was uneventful and complete.

Fixture head impressions were taken 2 months later and two Procera (Nobel Biocare, Gothenburg, Sweden) titanium custom abutments constructed. A 3-unit ceramo-metal bridge was constructed and cemented over these with a temporary cement (Improv, Nobel Biocare, Gothenburg, Sweden) (Figs 5, 6).

\section{DISCUSSION}

Implant body fracture is a rare complication of implant treatment occurring in only about $0.6 \%$ of fixtures. An in vivo study analysing the mechanism of fracture of implants concluded that fatigue stress fracture occurs with repeated loading. ${ }^{4}$ In this case the re-cementation of the post crown at UR3 (13) may well have altered the occlusion causing excessive loading of the implant bridge resulting in a fatigue stress fracture. Rangert et al. ${ }^{5}$ found that partial bridges with cantilever designs accounted for $83 \%$ of implant fractures and that 90\% of fractures occurred in the posterior regions. It has been shown in animal studies that craterlike bone loss will occur around implants with excessive dynamic loading ${ }^{6}$ and this loss of bone support will allow flexing of an implant under loading and possibly contribute to a fatigue stress fracture. In the past-prosthetic screw loosening sometimes leading to screw fracture has occurred $^{7}$ when occlusal overload is present but improvements to screw designs, allowing higher preloads has made screw loosening much less common. However this may perhaps lead to failure elsewhere in the system such as fracture of the body of the implant. Such fractures can be difficult to manage as patients invariably expect a fixed solution and therefore will not accept a removable partial denture. Care must be taken during treatment planning to minimise the risk of implant body fracture by designing a prosthesis, which allows for axial loading where possible and a favourable distribution of
Fig. 4 Guide pin in fixture fragment during surgery to aid implant placement

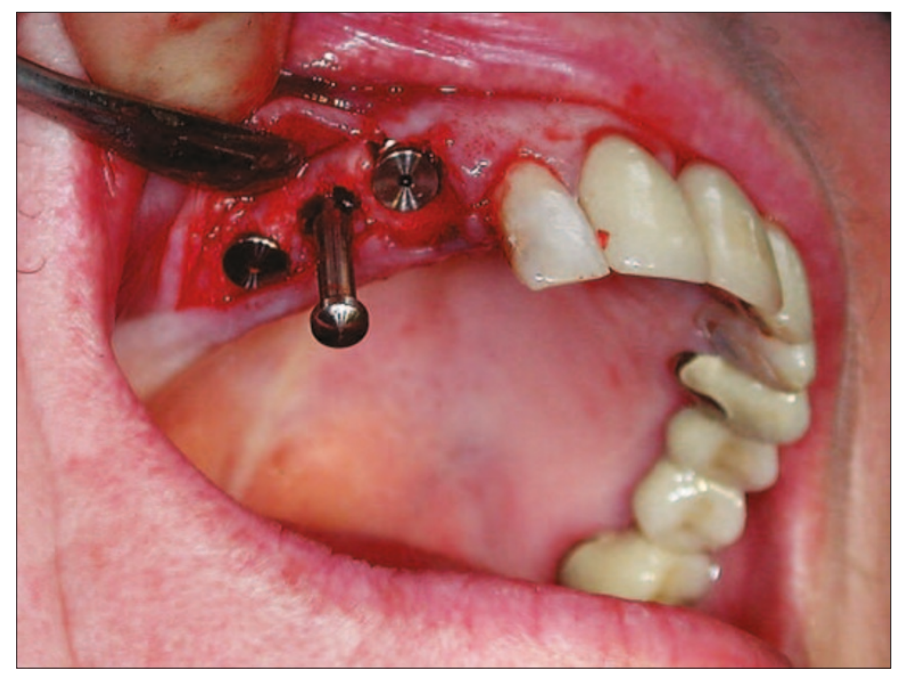


Fig. 5 Procera titanium abutments

Fig. 6 Final bridge in place
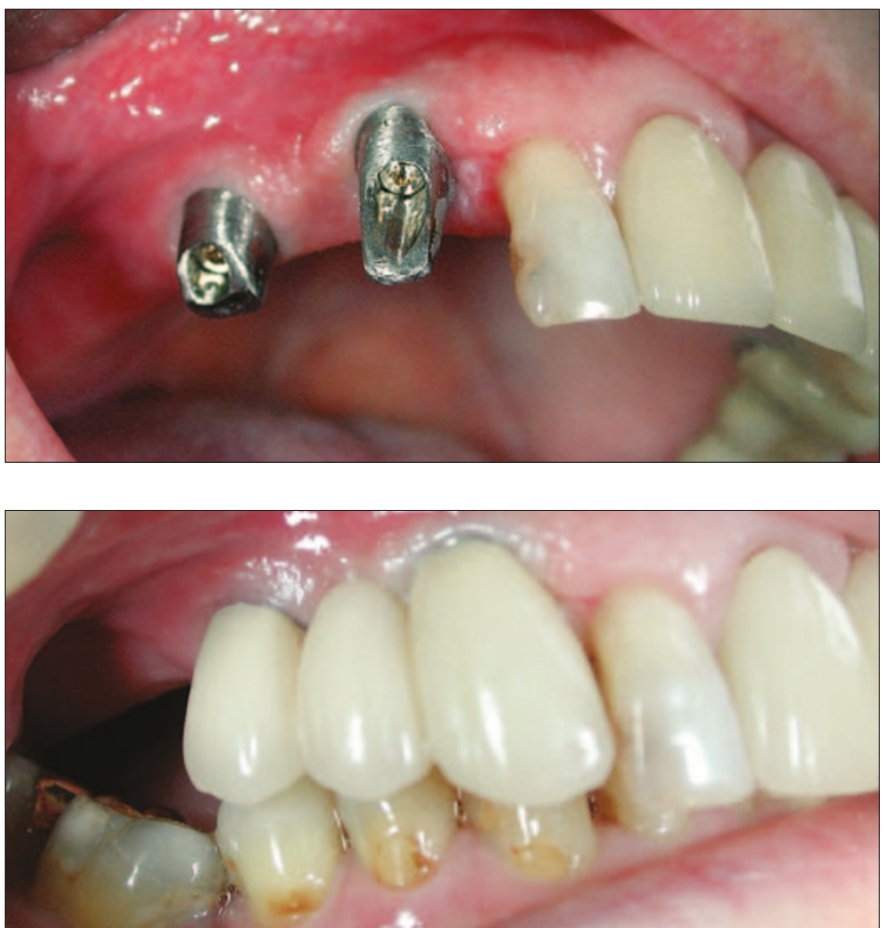

1. Adell R, Lekholm U, Rockler B, Branemark PI. A 15-year study of osseointegrated implants in the treatment of the edentulous jaw. Int J Oral Surg 1981; 10: $387-416$

2. Adell R, Eriksson B, Lekholm U, Branemark PI, Jemt T. Long-term follow-up study of osseointegrated implants in the treatment of totally edentulous jaws. Int J Oral Maxillofac Implants 1990; 5: 347-359.

3. Eckert SE, Meraw S J, Cal E, Ow R K. Analysis of incidence and associated factors with fractured implants: a retrospective study. Int J Oral Maxillofac Implants 2000; 15: 662-667.

4. Morgan J, James D, Pillar M. Fractures of the fixture component of osseointegrated implant. Int J Oral and Maxillofac Implants 1993; 8: 409-414.

5. Rangert B, Krogh P H, Langer B, Van Roekel N. Bending overload and implant fracture: retrospective clinical analysis. Int J Oral Maxillofac Implants 1995; 10: 326-334.

6. Duyck J, Ronold H J, Van Oosterwyck H, Naert I, Vander Sloten J, Ellingsen J E. The influence of static and dynamic loading on marginal bone reactions around osseointegrated implants: an animal experimental study. Clin Oral Implants Res 2001; 12: 207-218.

7. Kallus T, Bessing C. Loose gold screws frequently occur in full-arch fixed prostheses supported by osseointegrated implants after 5 years. Int J Oral Maxillofac Implants 1994; 9:169-178. occlusal forces. The avoidance of a cantilever design in partial cases, particularly in the posterior regions may well be advised. The periodontal ligament supporting a natural tooth will allow for movement of the tooth under loading and this will not occur when an implant is loaded as it is in direct apposition to bone, this may result in excessive loading of an implant in function and therefore must be avoided. Fracture of the body of an implant is a major cause of late failure $^{3}$ which may well be avoidable with careful planning.

The author thanks Dr Giles Davies for his help in carrying out the prosthetic treatment and Noble Biocare who kindly provided the fixtures and components.

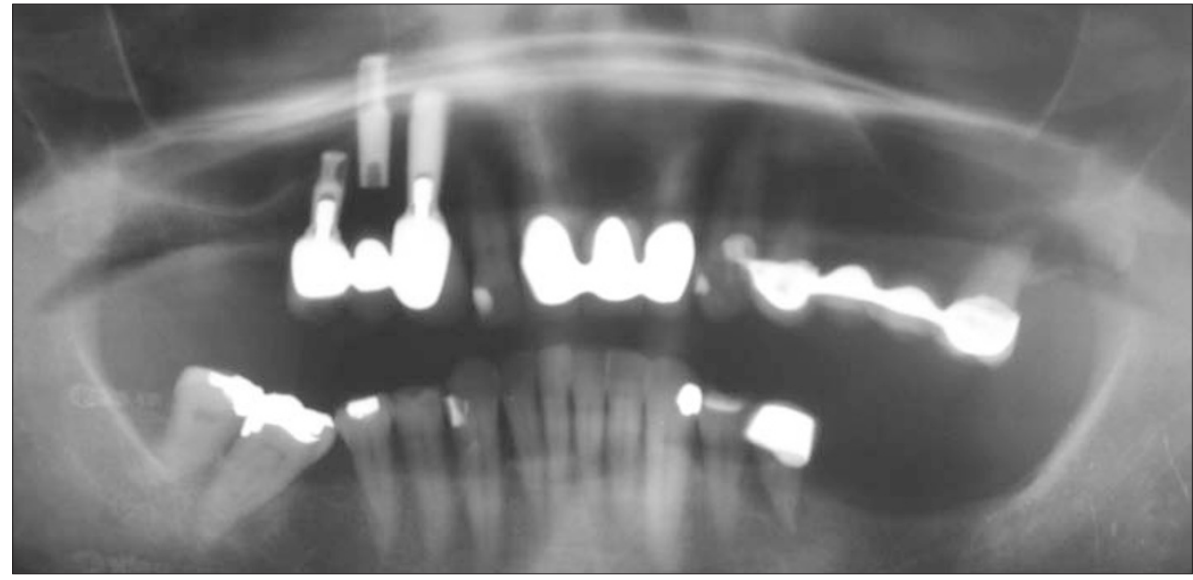

Fig. 7 Post treatment panoral radiograph 